\title{
Nematic Ferromagnetism on the Lieb lattice
}

\author{
CHEN Ke-Ji and ZHANG We* \\ Department of Physics, Renmin University of China, Beijing 100872, People's Republic of China and \\ Beijing Key Laboratory of Opto-electronic Functional Materials and Micro-nano Devices, \\ Renmin University of China, Beijing 100872, People's Republic of China
}

\begin{abstract}
We discuss the properties of ferromagnetic orders on the Lieb lattice and show that a symmetry protected quadratic-flat band crossing point will dramatically affect the magnetic ordering. In the presence of a weak on-site repulsive interaction, the ground state is a nematic ferromagnetic order with simultaneous broken of time-reversal and rotational symmetries. When the interaction strength increases, the rotational symmetry will restore at a critical value, and the system enters a conventional ferromagnetic regime. The mean-field transition temperatures for both the nematic and conventional ferromagnetic phases are in the order of interaction. This observation suggests that these magnetic orders have the potential to be realized and detected in cold atomic systems within realistic experimental conditions.
\end{abstract}

PACS numbers: 03.75.Ss, 37.10.Jk, 05.30.Fk

The investigation on ferromagnetism is one of the central topics in condensed matter physics, and has attracted attention for nearly a century since the early age of quantum theory. Heisenberg showed that a system of localized spins would favor a fully polarized state by gaining exchange energy. [1] However, the same argument can not be simply applied in itinerant fermions, where the kinetic energy of the underlying system has to be considered on an equal footing with the interaction effect.

The discussion on the stability of itinerant ferromagnetism in a lattice system dates back to 1960's. Thouless [2] and Nagaoka [3] pointed out that a ferromagnetic ground state can be stabilized in any finite bipartite lattice with an infinite on-site repulsive interaction. Lieb showed that the stable region of ferromagnetism can be extended to arbitrary repulsive interaction, provided that the number of sites are different for the composite sublattices. 4] The key ingredient in Lieb's argument is the existence of a non-dispersive, or equivalently flat, band. When the flat band is partially filled, fermions tend to be spin polarized to minimize the interaction energy, without paying any cost in the kinetic energy. In other words, since states in the flat band consist of localized Wannier functions, the ferromagnetic states can benefit from the exchange interaction as pointed out by Heisenberg. Subsequent studies confirm the stability of ferromagnetism in various models [5] 10, and generalize the idea to nearly-flat-band cases [11. Experimental realization of the (nearly) flat-band ferromagnetism has been proposed in a class of physical systems including atomic quantum wires [12, quantum-dot super-lattices [13], and organic polymers [14].

Rapid progress in cold atom experiments has paved a new route towards the exploration of ferromagnetism in itinerant fermions. Thank for the extraordinary controllability of lattice potentials and interaction, several

\footnotetext{
*Electronic address: wzhangl@ruc.edu.cn
}

proposals have been given to realize (nearly) flat-band ferromagnetism [15 17. In particular, Noda et al. investigated two-component cold fermions loaded into a twodimensional (2D) generalized Lieb lattice, and suggested that a ferromagnetic order can be stabilized in a wide parameter region. [16] The 2D generalized Lieb lattice consists of two square lattices (sublattice A and B), and has three sites per unit cell, as depicted in Fig.1(a). With only nearest-neighbor hopping, the lattice topology supports a flat band in the middle of two dispersive bands, hence can stabilize ferromagnetism when it is partially filled.

Apparently, a band structure containing only one single flat band does not exist in any realistic physical system. In all proposed model lattices for flat-band ferromagnetism, the flat band is always associated with dispersive bands, and the effect of their accompany is not fully understood. As a typical example, if the two composite sublattices of the Lieb lattice have the same depth, the flat band degenerates with the two linearly dispersive bands at the $\mathbf{M}=(\pi / d, \pi / d)$ point. On the other hand, in general cases where the depth of sublattice B is shifted from that of sublattice A by an amount of $V_{b}$, the flat band touches only one of the two dispersive bands at the M-point, as illustrated in Fig. 1(c), leading to a quadratic-flat band crossing point (QFBCP).

In this manuscript, we show that the ferromagnetic order is dramatically affected by the existence of the QF$\mathrm{BCP}$. In the non-interacting level, the QFBCP is protected by the time reversal (TR) and $C_{4}$ rotational symmetries. When a repulsive on-site interaction is present, since the density of state of the flat band is singular, the QFBCP becomes marginally unstable, leading to a spontaneous broken of the TR and/or $C_{4}$ rotational symmetries. At half filling, we find that in the weak coupling limit the ground state is a nematic ferromagnetic (NFM) order, where the spontaneous magnetization on sites 2 and 3 are different such that the $C_{4}$ rotational symmetry of the underlying lattice is broken down to $C_{2}$. The $C_{4}$ rotational symmetry will restore with increasing interac- 
(a)

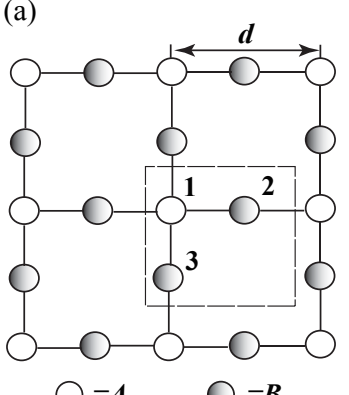

(b)

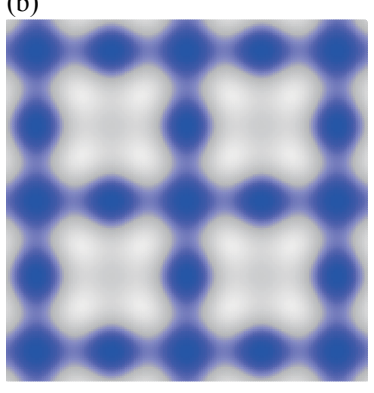

(c)
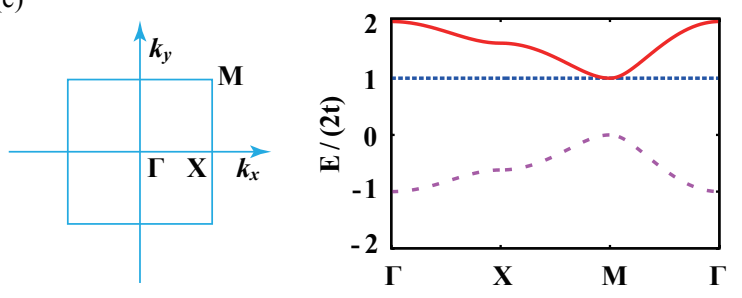

FIG. 1: (Color online) (a) 2D Lieb lattice consisting of two square sublattices $\mathrm{A}$ and $\mathrm{B}$. There are three sites $(1,2,3)$ within a unit cell (dotted square). (b) The Lieb lattice can be realized by arranging three square optical lattices. Here, a typical example with $V_{10}=V_{20}=2 V_{30}$ (see text) is shown to demonstrate the case of $V_{b} /(2 t)=0$. (c) Band structure of the Lieb lattice with $V_{b} /(2 t)=1$. Note that the flat band degenerates with the upper dispersive band at the M-point, leading to a quadratic-flat band crossing point.

tion via a second or first order phase transition, depending on the value of $V_{b}$. Within a mean-field calculation, we further map out the phase diagram, and identify three phases including: (i) a semimetal with NFM order; (ii) a band insulator with NFM order, and (iii) a band insulator with conventional FM order. These magnetic orders have the potential to be realized and detected in cold fermions loaded in optical lattices.

We consider spin $1 / 2$ fermions loaded in a $2 \mathrm{D}$ Lieb lattice

$$
\begin{aligned}
H= & -t \sum_{<i, j>, \alpha} c_{i \alpha}^{\dagger} c_{j \alpha}+V_{b} \sum_{i \in B} n_{i} \\
& +U_{A} \sum_{i \in A} n_{i \uparrow} n_{i \downarrow}+U_{B} \sum_{i \in B} n_{i \uparrow} n_{i \downarrow}
\end{aligned}
$$

where $t$ is the nearest neighbor hopping matrix, $c_{i \alpha}^{\dagger}\left(c_{i \alpha}\right)$ is the creation (annihilation) operator for fermion on site $i$ with spin $\alpha, V_{b} \equiv \epsilon_{B}-\epsilon_{A}$ is the chemical potential offset for the sublattice B (i.e., the relative shift of the two sublattices), $\epsilon_{i=A, B}$ describes an energy of each lattice site, and $U_{i=A, B}$ is the on-site interaction. In the context of cold atoms, a Lieb lattice with lattice parameter $d$ can be realized by arranging three square optical lattices with $V_{1}=V_{10}\left[\sin ^{2}(2 \pi x / d)+\sin ^{2}(2 \pi y / d)\right]$, $V_{2}=V_{20}\left[\sin ^{2}(\pi x / d+\pi)+\sin ^{2}(\pi y / d+\pi)\right]$, and $V_{3}=$
$V_{30}\left[\cos ^{2}(\pi x / d+\pi y / d)+\cos ^{2}(\pi x / d-\pi y / d)\right]$. In this configuration, the potential depths for sublattices $\mathrm{A}$ and $\mathrm{B}$ are $h_{A}=V_{10}+V_{20} / 2-V_{30}$ and $h_{B}=V_{10}-V_{20} / 2+V_{30}$, respectively, and can be controlled independently via suitable combination of laser intensities [18]. For an optical lattice given by the above configuration, parameters of Eq. (1) can be attained by using harmonic approximation where the Wannier function on a lattice site is approximated by the ground state wavefunction of a harmonic oscillator [19. Under this approximation, the on-site interaction can be rewritten as $U_{i} / E_{\mathbf{r}}=\left(2 h_{i} / \pi E_{\mathbf{r}}\right)^{1 / 2} a_{s} / a_{z}$, where the recoil energy $E_{\mathbf{r}} \equiv \pi^{2} \hbar^{2} /\left(2 m a^{2}\right)$ is used as the energy unit and $a_{z}$ is the characteristic length of $z$-axis trapping potential. Similarly, $t$ and $V_{b}$ can also be obtained analytically. According to the calculation above, this arrangement offers the ability to tune the hopping matrix $t$ and the potential offset $V_{b}$. A repulsive interaction $U_{i}>0$ can be achieved and varied via an adiabatic ramping to the upper branch on the BEC side of a Feshbach resonance [20].

In the non-interacting case, the Hamiltonian can be diagonalized in momentum space, leading to a band structure consisting of three bands as shown in Fig. 1(c). One of the three bands is completely flat, as required by the bipartiteness. The flat band has Bloch wavefunction $\propto\left[0,-\cos \left(k_{x} a\right), \cos \left(k_{y} a\right)\right]$ on the three sites within a unit cell, indicating the presence of local Wannier functions residing on sublattice $\mathrm{B}$ and having opposite amplitudes between sites 2 and 3 . When the chemical potential offset $V_{b}=0$, the three bands are degenerate at the $\mathbf{M}=(\pi / d, \pi / d)$ point, where the two dispersive bands linearly intersect with the flat band. In general cases of $V_{b} \neq 0$, depending on the sign of $V_{b}$, one of the two massive bands breaks the M-point degeneracy, and the flat band only touches the other dispersive band as illustrated in Fig. 1(c). As a consequence, the M-point becomes a quadratic-flat band crossing point (QFBCP). Around this point, the effective two-band Hamiltonian reads

$$
H_{0}^{\mathrm{eff}}=\frac{2 t^{2}}{V_{b}}\left(\begin{array}{cc}
|\delta \mathbf{k}|^{2} & \delta \mathbf{k}_{+}^{2} \\
\delta \mathbf{k}_{-}^{2} & |\delta \mathbf{k}|^{2}
\end{array}\right)+\mathcal{O}\left(\delta \mathbf{k}^{4}\right)
$$

where $\delta_{\mathbf{k}}=\mathbf{k}-\mathbf{M}$ and $\delta \mathbf{k}_{ \pm}=\delta k_{x} \pm i \delta k_{y}$. From this effective Hamiltonian, it is clear that the cases of positive and negative chemical potential offset $V_{b}$ are equivalent via a particle-hole transformation. Thus, we focus on systems with $V_{b}>0$ without loss of generality in the following discussion.

The presence of a QFBCP at the M-point is the central feature of the Lieb lattice. This band crossing point (BCP) is protected by the TR and $C_{4}$ rotational symmetries in the non-interacting case, and is characterized with a nontrivial topological index $2 \pi$. Such a putative topologically stable BCP becomes marginally unstable against infinitesimal repulsive interaction [21, leading to a spontaneous broken of TR and/or $C_{4}$ rotational symmetries, which drives the system towards a magnetic and/or nematic phase. Besides, since the BCP consists 

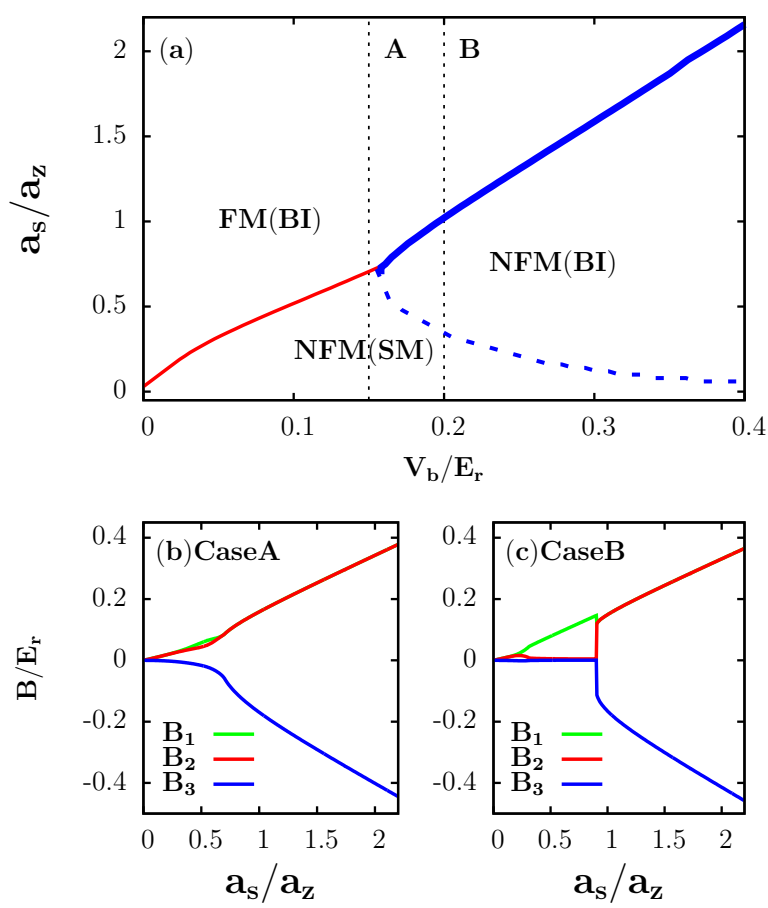

FIG. 2: (Color online) (a) Zero temperature phase diagram for spin $1 / 2$ Fermi system on the Lieb lattice at half filling. The onsite magnetization within sublattice B preserves the lattice symmetry within the FM phase, and becomes nonuniform in the NFM regime. The NFM phase is favored for weak interaction, and is separated from the FM regime via a second order (thin solid) or first order (thick solid) transition. Within the NFM regime, the system is a semimetal (SM) for small $a_{s} / a_{z}$ and $V_{b} / E_{\mathbf{r}}$, and becomes a band insulator (BI) with increasing $a_{s} / a_{z}$ and $V_{b} / E_{\mathbf{r}}$. For two typical values of $V_{b} / E_{\mathbf{r}}=0.15$ and 0.2 , the magnetization on the three sites are shown in (b) and (c), respectively. Here, we fixed $V_{10}=0.5 E_{\mathbf{r}}$ and $V_{30}=0.1 E_{\mathbf{r}}$.

of a non-dispersive band, the infinite density of state allows the possibility of filling the higher momentum states with one single spin species without gaining any kinetic energy. As a consequence, if the on-site interaction $U_{i}$ is repulsive, the system could easily favor a ferromagnetic phase for filling factors between $1 / 3$ and $2 / 3$.

Next, we focus on the case of repulsive Hubbard $U_{i}>0$ at half filling, and investigate the possibility for a stable ferromagnetic order. Within a mean-field (MF) level, the magnetic order is characterized by on-site magnetization $\mathbf{B}_{i=1,2,3} \equiv-2 U_{i}\left\langle\mathbf{S}_{i}\right\rangle /(3 \hbar)$, where $\mathbf{S}_{i}$ is the spin operator on site $i$. The momentum-space Hamiltonian takes the form

$$
H_{\mathbf{k}}^{\mathrm{MF}}=\left(\begin{array}{ccc}
\mathbf{B}_{1} \cdot \vec{\sigma} & -2 t \cos k_{x} \cdot \mathbb{1} & -2 t \cos k_{y} \cdot \mathbb{1} \\
& \mathbf{B}_{2} \cdot \vec{\sigma}+V_{b} \cdot \mathbb{1} & 0 \\
& & \mathbf{B}_{3} \cdot \vec{\sigma}+V_{b} \cdot \mathbb{1}
\end{array}\right),
$$

where $\vec{\sigma}=\left(\sigma_{x}, \sigma_{y}, \sigma_{z}\right)$ represents Pauli matrices, $\mathbb{1}$ denotes identity in the spin space, and the lower offdiagonal elements are filled to guarantee the Hermiticity of the Hamiltonian. The six energy bands of this Hamiltonian are occupied to half filling, leading to the energy functional

$$
\begin{aligned}
\mathcal{E}= & \sum_{(\mathbf{k}, j) \in \Gamma} E_{j}(\mathbf{k})+\frac{N_{0}}{2 U_{A}} B_{1}^{2}+\frac{N_{0}}{2 U_{B}}\left(B_{2}^{2}+B_{3}^{2}\right) \\
& +\frac{1}{6} N_{0} U_{A}+\frac{1}{3} N_{0} U_{B},
\end{aligned}
$$

where the summation over $(\mathbf{k}, j)$ is restricted to the set of occupied Bloch states $\Gamma$ with band index $j$, and $N_{0}$ denotes the total number of unit cells.

To study the possibility of nematic magnetic order with both TR and $C_{4}$ rotational symmetries broken, we allow the magnetization on sublattice $B$ can be different for sites 2 and 3, and map out the zero-temperature phase diagram as shown in Fig. 22(a) by minimizing the energy functional. We find that the optimized magnetization $\mathbf{B}_{i=1,2,3}$ are always along the $z$-axis, hence we consider only axial magnetic order in the following discussion.

In the weakly interacting limit, the nematic ferromagnetic phase (NFM) is always favorable with an exponentially small magnetization difference. When $a_{s} / a_{z}$ is increasing from the weakly interacting limit, the NFM phase remains stable up to a critical value $\left(a_{s} / a_{z}\right)_{c}$, above which the $C_{4}$ rotational symmetry restores and the system enters the FM regime. The order of the NFM-FM phase transition depends on the value of $V_{b}$. For $V_{b}$ below a critical value $V_{c} / E_{\mathbf{r}} \approx 0.156$, the NFM-FM transition is of the second order, as identified by the condition $V_{b}=B_{1 z}-B_{2 z}$ and depicted by a thin solid line in Fig. 2(a). By increasing $V_{b}>V_{c}$, the NFM-FM phase boundary becomes of the first order [thick solid line Fig. 2(a)], resulting from the competition between the corresponding metastable states.

Within the NFM phase, the $C_{4}$ rotational symmetry is spontaneous broken down to $C_{2}$ by splitting the QFBCP into two Dirac points located along the direction of one of the principal axes for weak interactions. The two Dirac points have the same Berry flux $\pi$, in clear contrast to the case of graphene where the two Dirac points have Berry fluxes $\pi$ and $-\pi$. In this case, the system is an anisotropic semimetal (SM) at half filling, with the Fermi surface shrinks to the two Dirac points as shown in Fig. 3(a). By increasing $a_{s} / a_{z}$, the two Dirac points move towards the boundary of the Brillouin zone, and eventually disappear when the magnetization difference $\delta=\left|B_{2 z}-B_{3 z}\right|$ between sites 2 and 3 exceeds the bandwidth of the first excited dispersive band. As a result, a full gap is open and the system becomes an anisotropic band insulator (BI) as depicted in Fig. 3(b). When the system enters the FM regime, the magnetization is large enough such that a finite gap is always present, and the system is a band insulator as shown in Fig. 3.(c). As the system enters the band insulator regime, being either a NFM or a FM state, we expect a quantum anomalous hall effect state can be stabilized provided a weak spin-orbit coupling 22 that supports a non-trivial Chern number 

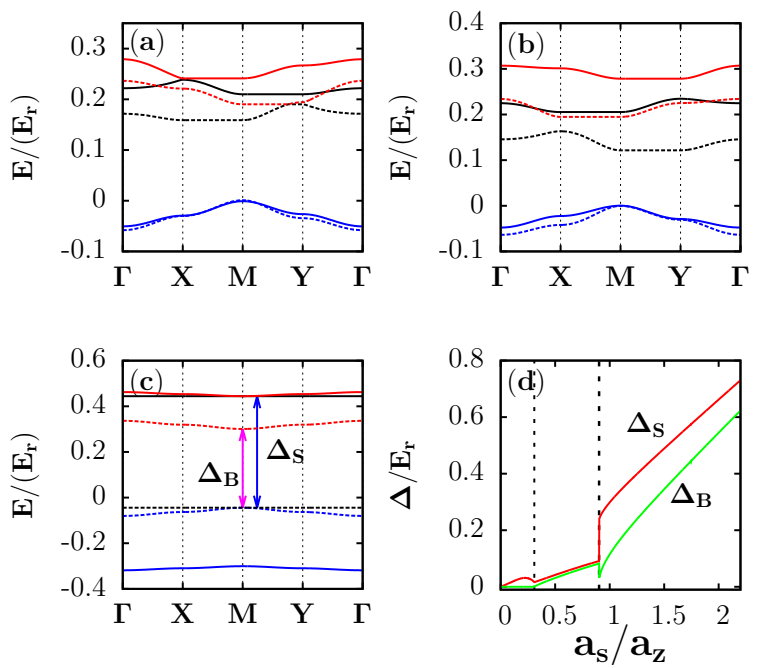

FIG. 3: (Color online) Band structure for (a) semimetal with NFM order, (b) band insulator with NFM order, and (c) band insulator with FM order. The solid (dashed) curves represent up (down) spin. These three cases are represented in the phase diagram Fig. 2(a), and correspond to $V_{b} / E_{\mathbf{r}}=0.2$, $a_{s} / a_{z}=0.3,0.5$ and 1.5 , respectively. The variation of $\Delta_{s}$ and $\Delta_{b}$ for $V_{b} / E_{\mathbf{r}}=0.2$ is shown in (d). Note that $\Delta_{s}$ is always in the order of the interaction. Here, $V_{10}=0.5 E_{\mathbf{r}}$ and $V_{30}=0.1 E_{\mathbf{r}}$ are fixed.

of the occupied band but does not close the band gap, as discussed in Ref. 23.

Note that in both the NFM and FM phases, the spin gap to create a minority spin $\Delta_{s}$ remains in the order of the interaction strength $U$ [See Fig. 3. d)]. Although the Mermin-Wigner theorem excludes the possibility of any $2 \mathrm{D}$ ferromagnetic order at finite temperature in the thermodynamic limit, the existence of such an order in a finite size system is perfectly allowed, provided that the coherence length $\xi$ is comparable or exceeding the system size $L$. Specifically, the coherence length of the magnetic order is $\xi \sim \hbar c \exp \left(\rho_{s} / T\right) /\left(k_{B} T\right)$, where $c$ is the spin-wave velocity at zero temperature, and the phase stiffness $\rho_{s}$ is in the same order of the MF transition temperature $T_{\mathrm{MF}}$ 24. Therefore, when the temperature goes well below $\Delta_{s}$, the coherence length can greatly exceed the lattice spacing such that domains with magnetic ordering can be formed. Since the energy gap $\Delta_{s}$ increases almost linearly with $U$, and the interaction can be tuned to be fairly large via an $s$-wave Feshbach resonance, the temperature required to observe the FM and NFM domains could be reachable within present technology.

In a realistic setup of cold atom experiment, a $2 \mathrm{D}$ geometry proposed in our manuscript is usually realized by a one-dimensional optical lattice or a pancakeshaped trap. The resulting trapping potential is highly anisotropic with typical trap size $L_{z} \sim \mu \mathrm{m}$ along the strongly confined axial direction and $L_{x y} \sim 10^{2} \mu \mathrm{m}$ in the quasi-2D radial plane. To incorporate the global trapping potential, one commonly used scheme is to implement the local density approximation (LDA) by introducing a position dependent chemical potential $\mu(\mathbf{r})$. The LDA is valid as the coherence length is much smaller than the radial trap size $\xi \ll L_{x y}$. [25] Besides, we also require the coherence length to be much greater than the lattice spacing $\xi \gg d$, such that FM or NFM ordered domains can be formed and resolved. Since the lattice spacing $d \sim 10^{2} \mathrm{~nm}$ is about 3 orders of magnitude smaller than $L_{x y}$, there is a large parameter window for the system to establish magnetic orders within LDA, where different phases coexist and form a ring structure.

The detection of the ferromagnetic orders can be implemented via an in-situ measurement 26, 27, which is able to extract single site density distribution for different spin species, and hence the local magnetization $B_{i} \propto$ $n_{i \uparrow}-n_{i \downarrow}$. If the system is prepared with equally populated two-component Fermi gas, we expect to resolve FM or NFM domains with opposite magnetizations. Another possible detection scheme is to measure the singleparticle dispersion with Bragg spectroscopy [28] or angleresolved photoemission spectroscopy (ARPES) [29].

In summary, we discuss the effect of a quadratic-flat band crossing point (QFBCP) on the ferromagnetic (FM) order. Taking the 2D Lieb lattice as an example, we show that the QFBCP is marginally unstable against infinitesimal repulsive interaction, given an infinite density of state of the flat band. In the weakly interacting limit, the ground state is a nematic ferromagnetic (NFM) order with time-reversal and rotational symmetries broken. Within the NFM regime, the spontaneous generated magnetizations are different on sublattice $\mathrm{B}$, and the QFBCP is broken into two Dirac points along one of the principal axes. In the strong coupling limit, the interaction $U$ becomes the only relevant energy scale, and a conventional ferromagnetic (FM) phase is favored. We then map out the zero-temperature phase diagram within a mean-field analysis, and identify three regions including a semimetal with NFM order, a band insulator with NFM order, and a band insulator with FM order. We point out that the spin gap for all three phases is in the same order of interaction, which can be tuned via a Feshbach resonance. Thus, we expect these magnetic phases can be realized in two-component Fermi gases loaded in optical lattices at experimentally reachable temperatures, and can be distinguished via a species selective in-situ measurement.

This work is supported by NSFC (10904172, 11274009), the Research Funds of Renmin University of China (14XNH061, 10XNL016), and the Program of State Key Laboratory of Quantum Optics and Quantum Optics Devices (KF201404). 
[1] Heisenberg W 1928 Z. Phys. 49619

[2] Thouless D J 1965 Proc. Phys. Soc. London 86893

[3] Nagaoka Y 1966 Phys. Rev. 147392

[4] Lieb E H 1989 Phys. Rev. Lett. 621201

[5] Mielke A 1991 J. Phys. A 24 L73

[6] Mielke A 1991 J. Phys. A 243311

[7] Mielke A 1992 J. Phys. A 254335

[8] Tasaki H 1992 Phys. Rev. Lett. 691608

[9] Tasaki H 1995 Phys. Rev. Lett. 754678

[10] Tanaka A and Tasaki H 2007 Phys. Rev. Lett. 98116402

[11] Tasaki H 1996 J. Stat. Phys. 84535

[12] Arita R et al 1998 Phys. Rev. B 57 R6854

[13] Tamura H, Shiraishi K, Kimura T, and Takayanagi H 2002 Phys. Rev. B 65085324

[14] Suwa Y, Arita R, Kuroki K, and Aoki H 2003 Phys. Rev. B 68174419

[15] Wang L, Dai X, Chen S, and Xie X. C. 2008 Phys. Rev. A $\mathbf{7 8} 023603$

[16] Noda K, Koga A, Kawakami N, and Pruschke T 2009 Phys. Rev. A $\mathbf{8 0} 063622$

[17] Zhang S, Hung H -H, and Wu C 2010 Phys. Rev. A 82
053618

[18] Goldman N, Urban D F, and Bercioux D 2011 Phys. Rev. A 83063601

[19] Jaksch D, Bruder C, Cirac J I, Gardiner C W, and Zoller P 1998 Phys. Rev. Lett. 813108

[20] Jo G -B et al 2009 Science $\mathbf{3 2 5} 1521$

[21] Sun K, Yao H, Fradkin E, and Kivelson S A 2009 Phys. Rev. Lett. 103046811

[22] Wu C -J, Ian M -S, and Zhou X -F 2011 Chin. Phys. Lett. 28097102

[23] Zhao A and Shen S -Q 2012 Phys. Rev. B 85085209

[24] Chakravarty S, Halperin B I, and Nelson D R 1988 Phys. Rev. Lett. 601057

[25] Stoof H T C, Gubbels K B, and Dickerscheid D B M 2009 Ultracold Quantum Fields (New York, Springer-Verlag)

[26] Gemelke N, Zhang X, Hung C -L, and Chin C $2009 \mathrm{Na}$ ture 460995

[27] Bakr W S et al 2010 Science 329547

[28] Ernst P T et al 2010 Nat. Phys. 656

[29] Stewart J T, Gaebler J P, and Jin D S 2008 Nature 454 744 